\title{
Relève, gouvernance de PME et comité consultatif : Jean-Yves Sarazin et la direction du Groupe DELOM ${ }^{1}$
}

\author{
Mircea-Gabriel Chirita, Claude Chapdelaine et Louis Jacques Filion \\ Chaire d'entrepreneuriat Rogers - J.A.-Bombardier \\ HEC Montréal
}

\section{INTRODUCTION}

Le Groupe DELOM représente un bel exemple de développement d'une PME familiale au travers de deux générations de dirigeants. La croissance de l'entreprise a été continue depuis sa fondation, voilà près de $\mathbf{5 0}$ ans.

Le Groupe DELOM représente un bel exemple de développement d'une PME familiale au travers de deux générations de dirigeants. La croissance de l'entreprise a été continue depuis sa fondation, voilà près de 50 ans. L'entreprise emploie 300 personnes. Son succès s'explique par plusieurs facteurs, mais en particulier par le type de gouvernance dont on a su doter l'entreprise. Au début des activités, les deux associés propriétaires s'occupaient de régler personnellement et dans les moindres détails le fonctionnement quotidien de leur entreprise. Mais la mise en place d'un comité consultatif a permis à la direction de l'entreprise de sortir de son

\section{LE GROUPE DELOM ET SON HISTOIRE}

Le Groupe DELOM est aujourd'hui composé d'entreprises autonomes qui proposent une gamme étendue de services complémentaires. Fondée en 1963, Delstar inc. est la plus ancienne unité du groupe. À l'origine, Delstar offrait principalement des services de réparation de moteurs électriques. Aujourd'hui, elle est spécialisée dans le service de réparation et de maintenance ainsi que dans le remplacement et la fourniture d'équipements rotatifs industriels. Les Ateliers Omega sont spécialisés dans la réparation mécanique, l'usinage et la fabrication/soudure ainsi que dans l'usinage d'équipements rotatifs.

DELOM Services Québec (anciennement: Services Électromécaniques Roberge inc.) œuvre dans isolement et de s'entourer d'une équipe d'experts possédant des compétences et expériences complémentaires qui sont venus dynamiser le processus stratégique de l'entreprise. L'évolution de la gamme de services offerts et l'augmentation graduelle du chiffre d'affaires du Groupe DELOM ne se sont pas produits par hasard. Ils sont la conséquence de changements dans la façon d'arrimer la gouvernance de l'entreprise. Cette étude porte sur les changements dans le système de gouvernance qui ont permis à cette PME de prendre son envol.

\section{L'évolution de la gamme de services offerts et l'augmentation graduelle du chiffre d'affaires du Groupe DELOM ne se sont pas produits par hasard. Ils sont la conséquence de changements dans la façon d'arrimer la gouvernance de l'entreprise.}

le domaine de la réparation des moteurs électriques depuis 1928, époque à laquelle elle portait le nom de « H. Roberge ». L'entreprise se joint au Groupe DELOM en 1997, et c'est en 2003 qu'elle se positionne stratégiquement dans l'Est du Québec pour se rapprocher de sa clientèle des régions de Québec, de Baie-Comeau et de Saguenay.

DELOM Solutions, c'est le regroupement des entreprises de services du Groupe DELOM: Delstar, Ateliers Omega et Roberge.

\subsection{Historique du Groupe DELOM}

Raymond Sarazin et son associé, Jacques Vallée, fondent officiellement Delstar en septembre 
1963 à Montréal-Nord. Ils lancent l'entreprise avec peu de capitaux. Raymond a 32 ans. Il possède certaines connaissances dans le domaine des moteurs électriques tandis que son associé est surtout orienté vers les ventes et le développement de la clientèle. Il s'agit d'un travail d'équipe et non pas celui d'une seule personne. Selon les dires de son fils, Jean-Yves, Raymond n'aurait pas lancé l'entreprise s'il avait été seul.

Lorsque son père fonde l'entreprise, Jean-Yves n'est alors âgé que de 12 ans. Il aura la chance de voir l'entreprise se développer sous ses yeux. Sur ce point, il dit : "Je me rendais souvent à l'entreprise pour y laver les camions et les planchers aussitôt que je finissais l'école et durant les fins de semaine. J'ai vu grandir l'entreprise. J'ai vu la machinerie que mon père a développée dans le sous-sol de notre maison et j'ai été témoin de ses nombreuses aventures. »

En fait, très jeune, Jean-Yves lie son destin à celui de l'entreprise. Il envisage d'abord étudier pour devenir ingénieur électrique parce qu'il croit que c'est important de maîtriser les connaissances de base sur ce que fait l'entreprise. Mais il se ravise. Comme il aspire à la direction de l'entreprise, il opte plutôt pour l'apprentissage de la gestion et entreprend un baccalauréat en administration à HEC Montréal.

En 1972, Jean-Yves est en troisième année de bac en administration lorsque son père et son associé décident de déménager Delstar. «La délocalisation de l'entreprise est devenue un de mes projets de fin d'études», nous confie JeanYves. Il doit trouver le terrain, faire l'aménagement intérieur, etc. Son devoir consiste à assurer l'entrée dans la nouvelle usine clés en main en août 1973, avec le défi de ne pas perdre d'employés.

Aussitôt diplômé, en 1973, Jean-Yves Sarazin commence à travailler en tant que contrôleur chez Delstar. Le défi l'attire même s'il aurait plutôt aimé travailler pour une grande entreprise, acquérir de l'expérience de gestion dans un contexte organisationnel plus grand et avoir l'occasion de voyager. Mais Delstar compte 30 employés et lui offre aussi plein de défis.
Deux ans plus tard, il relève un autre défi et fonde une nouvelle entreprise, Ateliers Oméga, un atelier mécanique spécialisé dans la réparation d'équipements mécaniques industriels.

J'ai fondé Omega en 1975 parce que, premièrement, je voulais avoir le défi de fonder aussi une entreprise. Deuxièmement, on donnait assez de travail à l'extérieur pour fonder notre propre machine shop. Troisièmement, on dépendait de quelqu'un d'autre pour une partie très importante de notre travail. On voulait mieux contrôler la qualité.

Jean-Yves détient $50 \%$ des actions des Ateliers Oméga, tandis que l'associé de son père détient les autres $50 \%$. Son frère vient le rejoindre et commence à travailler pour les Ateliers Oméga.

En 1978, Jean-Yves commence à prendre plus de place dans Delstar et réussit à obtenir de nouveaux clients. Ceci attire le mécontentement de l'associé de son père qui se retire sous prétexte de maladie et Raymond Sarazin rachète ses actions. Pourtant, l'associé agit dans l'ombre avec les autres cadres de direction pour lancer une compagnie concurrente. En découvrant les intrigues, grâce à une secrétaire vigilante, les Sarazin se voient forcés de licencier leurs cadres de direction. Sur ce point, l'extrait suivant est clair : «En entrant un matin de mai 1978, il y avait une équipe de gestion et, à $18 \mathrm{~h}$ le même jour, il n'y restait que deux personnes : mon père et moi. Je me suis retrouvé avec des employés dans l'atelier et des investissements sans plus personne à la gestion. Cela a été une période difficile! De bons souvenirs aujourd'hui, mais sur le coup, je me demandais si je ne venais pas de couper les pattes du temple parce qu'en même temps mon père commençait à se retirer tranquillement! Cette expérience m'a aidé ensuite au niveau de ma gestion d'employés. »

Ce fut une épreuve dure pour Jean-Yves, maintenant obligé de jouer plusieurs rôles afin d'assurer le bon fonctionnement de l'entreprise au niveau de la gestion.

Après ces départs, $\mathrm{j}$ 'avais toutes les jobs que je voulais: contremaître, acheteur, vendeur, relations avec les clients sur la 
route. L'associé de mon père étant parti, je devais m'occuper de ses clients, dont $80 \%$ de gros clients. Chercher un vendeur n'aurait pas fonctionné. Je devais d'abord faire la transition avec les clients et ensuite les déléguer, ce que j'ai commencé à faire dans les années 1980.

Après avoir occupé les principales fonctions au sein de l'entreprise, Jean-Yves devient président-directeur général de Delstar et d'Omega en 1986. Son père lui laisse la place et se retire des affaires. Jean-Yves décide alors de fusionner les deux entreprises et de faire équipe avec son frère qui travaillait au sein des Ateliers Omega. C'est ainsi que le groupe DELOM (la combinaison des trois premières lettres de Delstar et des deux premières lettres d'Omega) vit le jour en 1987. En 1996, le Groupe DELOM vivra une forte croissance suite à l'achat des Ateliers Roberge, entreprise située à Québec et qui deviendra DELOM Services Québec. Il s'en suit l'achat des Ateliers Wood de Baie-Comeau, qui étaient en situation de cessation de paiement. Cette entreprise s'appelle maintenant DELOM Services Baie-Comeau.

Il existe maintenant aussi d'autres divisions qui ont été mises sur pied: Delstar-Énergie, une filiale de Delstar spécialisé dans la réfection et la fabrication de bobines d'alternateurs hydroélectriques. Cette entreprise a très peu de clients, mais de gros clients tels Allstom et Hydro Québec. Tosbec, une autre filiale de Delstar, créée en 1996 pour la distribution exclusive de moteurs Toshiba au Québec : «Tosbec» pour Toshiba Québec.

\section{RELÈVE, STRATÉGIE ET COMITÉ CONSULTATIF}

\subsection{La relève et la mise en place d'un conseil} d'administration (CA)

Deux ans après avoir terminé son baccalauréat en gestion à HEC Montréal, en 1975, Jean-Yves manifeste son intérêt pour

l'implantation d'un conseil d'administration comme outil de gouvernance pour mieux répondre aux défis d'une PME en croissance telle que Delstar.

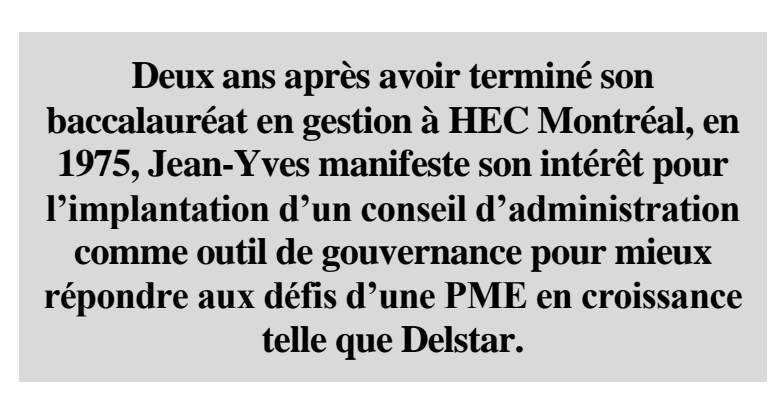

En 1986, lorsque Jean-Yves devient PDG, les deux entreprises combinées, soit Delstar et Omega, emploient 60 personnes et leur chiffre d'affaires s'élève à 6 millions \$. En 2010, le Groupe DELOM a un chiffre d'affaires de près de 50 millions $\$$ et compte près de 300 employés.

\subsection{La troisième génération de dirigeants}

En mai 2008, l'entreprise passe à une troisième génération de dirigeants. Cette année-là, JeanYves Sarazin cède la présidence et la direction générale du Groupe DELOM à Mario Montpetit, ingénieur mécanique, diplômé de l'Université de Sherbrooke. Mario travaille pour le groupe depuis près de 20 ans. Jean-Yves continuera d'être présent au sein du groupe en tant que président du Conseil d'administration.

Préparer la relève est un sujet majeur qui a été au cœur des préoccupations de Jean-Yves Sarazin pendant des années. Il travaille d'abord avec son frère pour mettre en place un système afin d'assurer la relève familiale du Groupe DELOM, mais les frères Sarazin finiront par envisager et finalement prendre une autre option.

Mon frère et moi avions à nous prononcer : «La propriété continuera-t-elle dans la famille ou pas? ». Entrevoyant une possibilité de chicane entre cousins et cousines, mon frère préférait que ce ne soit pas le cas. $\mathrm{Si}$ une offre ou quoi que ce soit arrivait demain matin, je la considérerais, en autant que la culture de l'entreprise soit respectée. On a mis cela clair pour le bien des actionnaires si jamais l'entreprise grandissait et qu'ils avaient la possibilité d'encaisser suite à une vente possible de l'entreprise.
Deux ans après avoir terminé son baccalauréat en gestion à HEC Montréal, en 1975, Jean-Yves manifeste son intérêt pour l'implantation d'un conseil d'administration comme outil de gouvernance pour mieux répondre aux défis d'une PME en croissance telle que Delstar. Mais son père, à l'instar de beaucoup d'entrepreneurs de cette époque, vit le conseil d'administration comme un frein, comme une instance dont il faut répondre et cela lui répugne «Non, les gens de l'extérieur ne viendront pas me dire quoi faire! ». 
Mais la mise en place d'un conseil d'administration deviendra réalité en 1986 lorsque Jean-Yves accèdera à la présidence de Delstar. Il assistera alors à des séminaires portant sur la manière de former, opérer et gérer un conseil d'administration. Il embauchera même un spécialiste pour l'aider à mettre les gens en place et à partir la première rencontre.

Le conseil d'administration est composé de six personnes : quatre personnes externes ainsi que Raymond et Jean-Yves Sarazin. Jean-Yves doit convaincre son père qu'il faut absolument qu'il y soit présent pour le transfert de la culture et pour faciliter le travail des membres du Conseil.

Mon père n'était pas content parce qu'il se demandait ce que ces quatre personnes feraient chez nous puisqu'ils ne connaissaient pas le domaine des moteurs. Au début, on y est allé doucement car il ne voulait même pas y être. Quand il a vu la dynamique, il était content. Ils m'ont fait sauver $40000 \$$, en partant, à la première réunion, en réaménageant nos prêts hypothécaires. Mon père y est resté pendant 10 ans.

Raymond Sarazin devient président du conseil d'administration, mais de facto, c'est Jean-Yves qui assume ce rôle. Les membres externes qui siègent au conseil d'administration de Delstar sont des spécialistes dotés de compétences complémentaires : un spécialiste en marketing et en vente, un expert en finances et un entrepreneur en ingénierie de la construction.

\section{La formation du conseil d'administration permet à Jean-Yves de s'entourer d'une équipe d'administrateurs dont l'expérience et les compétences respectives complètent bien les siennes. Les administrateurs sont là pour conseiller et aider Jean-Yves plutôt que pour remettre en question sa gestion.}

La formation du conseil d'administration permet à Jean-Yves de s'entourer d'une équipe d'administrateurs dont l'expérience et les compétences respectives complètent bien les siennes. Les administrateurs sont là pour conseiller et aider
Jean-Yves plutôt que pour remettre en question sa gestion. Le fait de s'être entouré d'une équipe compétente vient accroître la crédibilité de l'entreprise et permet à Jean-Yves de bénéficier d'un réseau de relations accru par celui des membres du conseil d'administration.

J'avais bien expliqué le but du Conseil, soit de m'aider à me valider. Ils étaient surtout là comme guides et non pas comme dirigeants. Ils n'avaient pas à trouver le président le lendemain matin puisqu'il était en place. Si mon père était décédé, je me serais senti drôlement mieux appuyé par eux. À 35 ans, être accompagné d'un conseil en place fait en sorte que tu auras le support financier grâce à la réputation des membres du conseil.

Selon Jean-Yves, en dehors de leurs compétences, les membres du conseil d'administration doivent avoir des expériences pertinentes dans le monde des PME. En fait, ce ne sont pas les administrateurs qui vont résoudre les problèmes de gestion, ils doivent plutôt savoir identifier ces problèmes et indiquer qui pourrait s'en occuper :

J'essaie de faire attention à ça lorsque je sélectionne des membres: en quoi a-t-il vécu quelque chose de semblable? Dans le fond, on partage le vécu. Comment peut-on partager un vécu qu'il n'a pas ? Si j'ai un problème de marketing, même si le gars autour de la table a beaucoup d'expérience en marketing, ce n'est pas lui qui pourra résoudre le problème. Par contre, il devrait pouvoir me pointer une firme capable de m'aider.

Le conseil d'administration de Delstar fonctionnait par mandats de deux ans à raison de quatre rencontres par année, une tous les trois mois.

\subsection{Mise en place d'un comité consultatif (CC)}

Dès son accession à la présidence de l'entreprise en 1986, Jean-Yves Sarazin met sur pied un conseil d'administration dans le but de se faire aider et accompagner dans la transition à la direction de l'entreprise. Le développement de 
l'entreprise lui tient à cœur et il compte opérer à partir d'un plan stratégique bien articulé. Il sait qu'il aura aussi besoin d'aide pour le formuler et éventuellement pour le mettre en œuvre. Selon lui, à cette époque, le statut de conseil d'administration se présentait comme une formalité juridique et comme la façon courante de bien arrimer la gouvernance d'une entreprise.

Avec le temps, la responsabilité légale associée au statut d'administrateur a évolué. En outre, le maintien d'un conseil d'administration engendrait des difficultés occasionnelles pour couvrir certains risques des administrateurs par des assurances appropriées. C'est pourquoi, après quatre ans de ce régime, soit en 1990, Jean-Yves décide de mettre en place une structure plus souple.

\section{C'est à ce moment que Jean-Yves Sarazin remplace le conseil d'administration par un Conseil Consultatif. Contrairement aux membres d'un conseil d'administration qui sont responsables légalement de tout ce que fait l'entreprise, les membres d'un Conseil Consultatif n'encourent aucune responsabilité légale. Ils agissent en tant qu'experts-conseils.}

C'est à ce moment que Jean-Yves Sarazin remplace le conseil d'administration par un Conseil Consultatif. Contrairement aux membres d'un conseil d'administration qui sont responsables légalement de tout ce que fait l'entreprise, les membres d'un Conseil Consultatif n'encourent aucune responsabilité légale. Ils agissent en tant qu'experts-conseils. Il revient à la direction d'entreprise de les écouter ou non, de mettre en œuvre ou non les décisions qui découlent de leurs recommandations. Toutefois, un rapport professionnel équivalent à celui assumé par l'ancien conseil d'administration est partagé par les membres qui le vivent comme tel.

Je continuerai avec les mêmes gens, sauf qu'ils ne seront pas administrateurs et qu'il ne sera pas question d'assurances ! En assistant au séminaire de Louis-Paul Malette, je m'étais aperçu qu'une PME n'avait pas besoin d'un conseil d'administration, mais d'un comité aviseur. On l'a appelé Comité consultatif externe.

En 2010, le Conseil Consultatif du Groupe DELOM compte 10 membres dont 3 membres externes, les directeurs de chacune des quatre entreprises du groupe, le PDG du groupe, le vice-président chargé du développement des affaires, Jean-Yves Sarazin et son frère. D'après Jean-Yves, le Conseil Consultatif doit représenter les quatre piliers de l'entreprise : le social, soit les ressources humaines, le juridique, le financier et la connaissance du marché. Les aspects juridiques du groupe ne demandent pas une attention particulière. Ainsi, Jean-Yves considère n'avoir besoin dans son comité que de trois membres externes ayant chacun des compétences distinctives dans les trois domaines de gestion clés de l'entreprise: ressources humaines, finances et ventes. Il convient de mentionner les deux principes qui guident JeanYves Sarazin dans la formation d'un Conseil Consultatif : pas de connaissances personnelles, ni de professionnels qui travaillent pour la compagnie. Il ne faut pas que les membres du Conseil Consultatif soient susceptibles de devenir des fournisseurs ou des consultants de l'entreprise. Le Conseil Consultatif se réunit cinq fois par année : une réunion tous les trois mois et une réunion spéciale une fois par année. Selon Jean-Yves, le Conseil Consultatif doit se réunir quatre fois par année quelle que soit la taille de l'entreprise.

Il faut quatre réunions par année au minimum. Ceux qui font une réunion à tous les mois finissent par avoir un comité de gestion. C'est une autre affaire. Un bon comité consultatif consiste à aider le dirigeant dans le développement de son entreprise.

Il arrive également que Jean-Yves rencontre individuellement les membres externes du Conseil Consultatif. La préparation pour une rencontre ne demande pas beaucoup de temps puisque chaque membre reçoit d'avance un procès-verbal comprenant deux pages, sauf pour des situations exceptionnelles, tel qu'un projet d'investissement. 


\subsection{Comité consultatif et réflexion stratégique}

Alors que les cadres de la direction assurent la gestion courante de l'entreprise, le rôle du Conseil Consultatif comporte un volet stratégique, car il accompagne le PDG dans les réflexions et questions complexes touchant l'évolution de son organisation. Ainsi, le plan stratégique du PDG est évalué et validé par le Conseil Consultatif. C'est cette dimension qui a fait en sorte que Jean-Yves a décidé de suivre des cours sur la réflexion stratégique, avant de mettre en place des outils de gouvernance, en particulier lors du remplacement du conseil d'administration par un Conseil Consultatif. Son premier plan stratégique a été plutôt une réflexion stratégique pour laquelle il demanda l'aide à un consultant, Gilles Charest :

«J'ai une petite hantise envers les consultants qui te la mettent sur ton bureau en disant que c'est ce qu'on doit faire! Je voudrais plutôt que tu me coaches dans cette réflexion stratégique! ».

Jean-Yves a travaillé avec Gilles Charest pendant plusieurs années afin de bien roder sa méthode de fonctionnement avec le Conseil Consultatif. En plus de cela, à partir de 1999, Jean-Yves utilise la méthodologie DEVCOM. Cette approche va l'aider à structurer d'une manière simple et efficace l'activité de son groupe :

En 1999, Guy Desmarais de DEVCOM nous a amené une technique nous permettant de trouver l'équilibre entre l'utilisation des chiffres et les autres objectifs à atteindre. Les chiffres ne nous conditionnent pas. Ils ont toujours été la résultante de quelque chose et non pas l'objectif à atteindre. Depuis, on utilise sensiblement la même méthode, notre meilleure méthode à date.

\subsection{Le Groupement des chefs d'entreprise du Québec}

Il convient de noter que le Conseil Consultatif n'est pas le seul outil à la portée d'un entrepreneur pour se faire accompagner et échanger des expériences vécues et du «comment faire». Le soutien peut également venir de la part d'un mentor, d'un coach, d'organismes communau- taires, de différents réseaux ou associations d'affaires. Aucun d'entre eux n'exclut un autre et chacun peut aider à sa façon l'entrepreneur et le propriétaire-dirigeant de PME à accomplir sa mission, sa vision et ses objectifs. Par exemple, un élément majeur l'ayant aidé à structurer, à comprendre et à développer la stratégie de son groupe a été l'implication de Jean-Yves Sarazin dans le Groupement des chefs d'entreprise du Québec. Il rejoint les rangs du Groupement des chefs d'entreprise en 1986 lorsqu'il devient PDG chez Delstar. Selon ses dires, il s'agit d'une véritable « école de chefs ».

Même en présence de gens des HEC, je peux dire qu'il m'a appris à devenir un chef. HEC Montréal m'a donné les connaissances et le Groupement m'a accompagné dans mon développement, dans la capitalisation de mon expérience. Il a été extrêmement important pour moi.

\section{Il convient de noter que le Conseil Consultatif n'est pas le seul outil à la portée d'un entrepreneur pour se faire accompagner et échanger des expériences vécues et du « comment faire ». Le soutien peut également venir de la part d'un mentor, d'un coach, d'organismes communautaires, de différents réseaux ou associations d'affaires.}

Jean-Yves apprécie surtout la formule des clubs $\mathrm{du}$ Groupement qui permet de partager ses problèmes et ses inquiétudes avec des gens qui ont vécu des expériences similaires.

Il y a rencontré son vice-président chargé du développement des affaires qui était un ancien entrepreneur. Son implication dans l'activité du Groupement fut grandissante: en 1994, il accepte le poste de secrétaire-trésorier et en 1999, il est nommé président du Groupement, mandat d'une durée d'une année.

\subsection{Pourquoi un Conseil Consultatif... l'avis de Jean-Yves Sarazin}

Il n'est pas rare que le PDG d'une PME soit l'homme-orchestre qui règle personnellement et dans les moindres détails la gestion courante de son entreprise, ce qui lui fait perdre, à long 
terme, une vision plus globale. Isolé, il tend à diriger tout personnellement plutôt que de discuter avec ses cadres d'avenues possibles, et ce, même s'il n'a pas toujours la compétence pour le faire.

C'est pourquoi, selon l'avis de Jean-Yves Sarazin, la formation d'un Conseil Consultatif permet aux entrepreneurs de sortir de leur isolement et d'avoir une place où ils peuvent confronter leurs orientations. Un Conseil Consultatif permet aussi de se remettre constamment en question en tant que dirigeant d'entreprise. Plus important que tout, un Conseil Consultatif force à réfléchir et à préparer la relève. La mission d'un Conseil Consultatif consiste à poser les vraies questions, à aller au fond des choses. Ce comité doit avoir l'autorité pour affirmer, par exemple, que le plan de succession envisagé par le fondateur manque de réalisme et qu'il doit être révisé de fond en comble.

Le conseil permet aussi de combler ses faiblesses. Un entrepreneur moins chevronné en finances pourra bénéficier des recommandations d'un membre du Conseil Consultatif qui est spécialiste dans ce domaine. De plus, un Conseil Consultatif demande de la préparation, ce qui déclenche un processus de réflexion: «En se préparant, souvent on corrige nous-mêmes la direction, sans plus avoir besoin de la rendre au Conseil. On réfléchit sur l'idée première, sur ce qui peut la modifier. » En d'autres mots, selon Jean-Yves Sarazin, «un Conseil Consultatif permet une belle discipline ».

\section{LES AVANTAGES DES INNOVATIONS EN MATIÈRE DE GOUVERNANCE}

3.1 Gouvernance de PME et comité consultatif : réflexions de Jean-Yves Sarazin

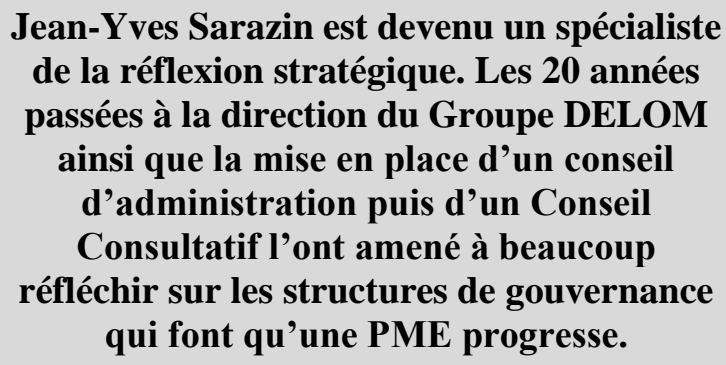

Jean-Yves Sarazin est devenu un spécialiste de la réflexion stratégique. Les 20 années passées à la direction du Groupe DELOM ainsi que la mise en place d'un conseil d'administration puis d'un Conseil Consultatif l'ont amené à beaucoup réfléchir sur les structures de gouvernance qui font qu'une PME progresse. Il a suivi des cours sur le sujet, travaillé avec des consultants experts en matière de gouvernance de PME, partagé des pensées avec de nombreux dirigeants de PME, en particulier depuis les années 2000, années où il a exercé des fonctions de direction au Groupement des Chefs d'entreprise du Québec. Il est intarissable sur la stratégie des PME ainsi que sur le rôle qu'un Conseil Consultatif peut jouer pour mieux orienter une entreprise. Le texte qui suit présente des extraits de ses réflexions sur le sujet.
Ces extraits portent sur les lignes directrices qui ont guidé Jean-Yves dans le fonctionnement d'un Conseil Consultatif et ses conseils pour les dirigeants de PME qui souhaitent mettre sur pied un tel outil de gouvernance.

\subsection{Les avantages d'un Conseil Consultatif}

Selon l'avis de Jean-Yves Sarazin, la formation d'un Conseil Consultatif permettrait aux dirigeants de PME de mieux définir leur vision et stratégie et d'avoir une place où l'on peut confronter nos orientations. Elle permettrait aussi d'être plus intrépide et d'essayer de nouvelles choses.

«Les premières années, le Conseil Consultatif m'a permis d'avoir la liberté d'essayer et de développer des choses pour lesquelles mon père aurait peut-être dit «non». Ce que j'aurais eu à dire parfois à mon père, par exemple: «Il faudrait au moins essayer...», je n'avais pas besoin de mettre de pression car le Conseil Consultatif disait : "Cela a de l'allure, on va l'essayer et tu nous reviendras avec un compte rendu plus tard! ». En me donnant leur accord, ils m'aidaient beaucoup. Mon père a bien aimé de son côté parce qu'il s'est aperçu qu'il n'avait pas besoin de me dire ce qu'il aurait eu à me dire; le Conseil Consultatif me le disait. » 
La formation d'un Conseil Consultatif permet également de combler leurs limites. Sur ce point, Jean-Yves Sarazin note: «Le Conseil Consultatif nous permet de compléter nos forces et faiblesses. Le dirigeant d'entreprise moins versé en finances choisira souvent quelqu'un en finances qui pourra lui suggérer d'aller voir un tel ou un tel lors de points de fiscalité ou d'achat de machinerie.» Il ajoute que : «Le Conseil Consultatif est un élément extrêmement important pour voir la philosophie en place au niveau de la culture de l'entreprise, de la façon de gérer les ressources humaines si on veut éviter un syndicat ou si on veut apprendre comment transiger s'il y en a un. »

Un autre avantage lié à la formation d'un Conseil Consultatif est de préparer la relève. Pour M. Jean-Yves Sarazin : «le Conseil Consultatif m'a beaucoup aidé à faire grandir les gens de la direction et à cibler la bonne relève. Je leur demande souvent ce qu'ils pensent des nouveaux. Leur opinion d'une rencontre d'une heure avec une personne peut être une impression du moment qui n'est pas son portrait, mais ils touchent parfois réellement le point d'évaluation de cette personne. »

Enfin, le Conseil Consultatif permet d'ajouter de la crédibilité à leur entreprise. Selon Jean-Yves Sarazin : «Le Conseil Consultatif a un impact énorme au niveau de nos relations avec nos banques et directeurs financiers, du fait qu'ils savent qu'un Conseil Consultatif administratif ou consultatif est en place. Cela m'a beaucoup aidé d'en avoir un.»Il ajoute que : «On est client d'une même banque depuis 45 ans. Je les ai encouragés à regarder s'il y avait un Conseil Consultatif en place chez leurs clients : «Vous ne devriez pas accepter d'avoir un client s'il n'a pas de Conseil Consultatif. Cela devrait faire partie de vos conditions de prêts. Ainsi, avant de tirer sur le prêt, j'irais voir le Conseil Consultatif que vous lui aurez demandé de mettre en place! C'est la plus grande sécurité. »

\subsection{L'importance d'un Conseil Consultatif}

Étant convaincu de l'importance d'un Conseil Consultatif et souhaitant partager son expérience, Jean-Yves siège sur plusieurs Conseils Consultatifs relevant d'entreprises très diffé- rentes en ce qui concerne leur taille et leur domaine d'activité. Même si le principe est le même, chaque Conseil Consultatif a sa spécificité, soit la préparation des chiffres est très détaillée, soit l'accent est mis sur la préparation de la relève ou l'accompagnement du nouveau PDG dans le développement de l'entreprise. Selon Jean-Yves, beaucoup de Conseil Consultatif sont trop concernés par les indicateurs chiffrés. Selon lui, ceci ne devrait pas constituer la préoccupation principale d'un Conseil Consultatif. À cet égard, Jean-Yves Sarazin dit que : «Les chiffres sont un portrait. On peut voir si ça va en haut ou en bas, on peut poser des questions, mais ils ne sont pas le but d'un Conseil Consultatif. La personne qui veut jaser de ses chiffres comptables est mieux de dépenser de l'argent avec un comptable externe pour passer à travers et lui demander ses suggestions. » Il note aussi que : «Le but principal d'un Conseil Consultatif concerne le développement de l'entreprise : marché, ressources humaines, équilibre, finances. Dans le fond, il faut faire grandir l'entreprise. »

Le fait d'opérer dans un Conseil Consultatif est très motivant pour Jean-Yves, car cela lui donne le sentiment d'aider ses confrères en partageant avec eux son expérience. Sur ce point, il dit que «La récompense de faire partie d'un Conseil Consultatif n'est pas dans la paie; on ne le fait pas pour elle. Les honoraires sont là pour apporter un petit peu de sérieux de la part des gens qui convoquent, pour que ce ne soit pas un club de rencontres sociales. La récompense est réellement le sentiment d'apporter quelque chose, d'aider, comme ta mission sociale, ton engagement à transférer aux autres ce que tu as eu la chance d'apprendre. »

Étant donné sa volonté de partager son vécu et accompagner les dirigeants de PME, Jean-Yves trouve moins stimulant le travail au sein des conseils de grandes entreprises. Ces dernières ont l'expertise qu'il leur faut et tout ce qui reste à faire est d'évaluer les divers indicateurs. Toutefois, le travail au sein des Conseils Consultatifs de petites entreprises lui parait plus incitatif et stimulant, car la direction a besoin d'astuces pour compenser certaines ressources insuffisantes. À cet égard, Jean-Yves Sarazin est 
clair en disant que : «Un gros conseil d'une grande entreprise où tout est superbement préparé, ce n'est pas le fun! Je ne suis pas sûr que je sois heureux au sein d'un conseil d'administration d'une grande entreprise où on fait la lecture de documents, puis on demande : « accepté ou refusé »? Les dossiers étant gros, je ne sais pas le fun que j'aurais parce que tout est tellement pensé d'avance. Des heures et des heures ont été mises, alors comment peut-on nous permettre, nous, en quelques minutes, de dire...? Je n'ai pas tripé nécessairement aux conseils, style de grandes entreprises. »

\subsection{La continuité}

Dans la même logique, Jean-Yves est motivé à accompagner les dirigeants de PME qui travaillent à la continuité de leur entreprise. On siège sur un Conseil Consultatif afin d'aider un dirigeant de PME à naviguer à travers les eaux troubles et à faire grandir son entreprise. Comme l'illustre le passage suivant : «Mon guide est la continuité de l'entreprise. Avant de faire partie d'un Conseil Consultatif, je veux savoir si la personne veut travailler à la continuité de son entreprise. Pour moi, il s'agit de la relève, du développement des affaires, de la finance. La décision de vendre peut arriver un moment donné mais si son but premier est de farder la mariée pour la vendre, je ne suis pas intéressé à en faire partie. Je suis motivé à travailler pour la faire grandir et...»

\subsection{Préparer la relève}

D'après Jean-Yves, le Conseil Consultatif a un rôle particulièrement important à jouer dans l'accompagnement du dirigeant de PME qui cède sa place à un membre de sa famille.

Le but du dirigeant de PME consiste à choisir le meilleur pour lui succéder. Il faut viser la continuité de l'entreprise. Le Conseil Consultatif est là pour assister le président dans le développement de l'entreprise et pour s'assurer qu'elle continuera. J'ai vu souvent des gens misant beaucoup sur les membres de la famille choisir le plus vieux quand ce ne devait pas être lui.

Ils doivent être conscients d'un phénomène que j'ai eu à vivre et que j'ai vu dans la PME. C'est une autre affaire dans la grande entreprise. La priorité d'avoir seulement la famille à la direction de l'entreprise éloigne de très bons candidats. Ce n'est pas facile, mais il faut s'assurer qu'il y ait de la place. Je connais une entreprise, entre autres, qui recommence tout le temps au niveau de la composition de l'équipe de direction parce qu'il est écrit sur le mur en entrant qu'elle est dédiée, bons, pas bons, aux membres de la famille. Si j'aspire à gravir les échelons et à occuper un poste de direction un jour, je n'irai pas travailler pour cette entreprise, sachant que j'y perdrais mon temps, qu'elle deviendrait un passage pour autre chose, un titre sur mon c.v. C'est dommage, car ce sont les employés qui te donnent toute ta légitimité. Ce devrait être un processus naturel.

Si le président qui forme le Conseil Consultatif vise à assurer la continuité de l'entreprise ou à mettre ses enfants en place, il y aura de petites discussions avant de dire comment le préparer, comment l'y amener. À la limite, si un président d'entreprise voulait mettre un membre de la famille en place et que cette personne n'est pas la bonne, par mon souci de continuer l'entreprise pour les employés, les actionnaires, j'essaierais probablement de discuter avec lui pour voir au juste si ce ne serait pas bon d'avoir un autre avis. Sinon, ces entreprises mourront, sans valeur le lendemain.

\section{NOTES}

${ }^{1}$ Cette étude de cas est publiée avec la permission du Centre de cas d'HEC Montréal.

${ }^{2}$ Président de Sociogest, Gilles Charest est un pionnier du développement organisationnel au Québec. Détenteur d'une maîtrise en administration des affaires (MBA), il œuvre depuis 1971 comme expert-conseil en management et en développement des organisations.

${ }^{3}$ DEVCOM est une approche de stratégie d'affaires qui favorise la concentration des énergies des dirigeants sur les activités essentielles des entreprises, la délégation des responsabilités et la reconnaissance de la contribution du personnel au succès des entreprises. La méthode a été mise au point pas Guy Desmarais, reconnu comme un gestionnaire hors pair par la communauté des affaires du Québec. Site : http://www.devcom.qc.ca/

${ }^{4}$ Fondé en 1974, le Groupement des chefs d'entreprise du Québec est un réseau d'entraide, composé exclusivement de 1500 chefs propriétaires et aspirants-chefs de PME, répartis dans plus de 200 clubs, dans toutes les régions du Québec, au Nouveau-Brunswick et en Belgique. Site: http://www.goupement.ca/ 


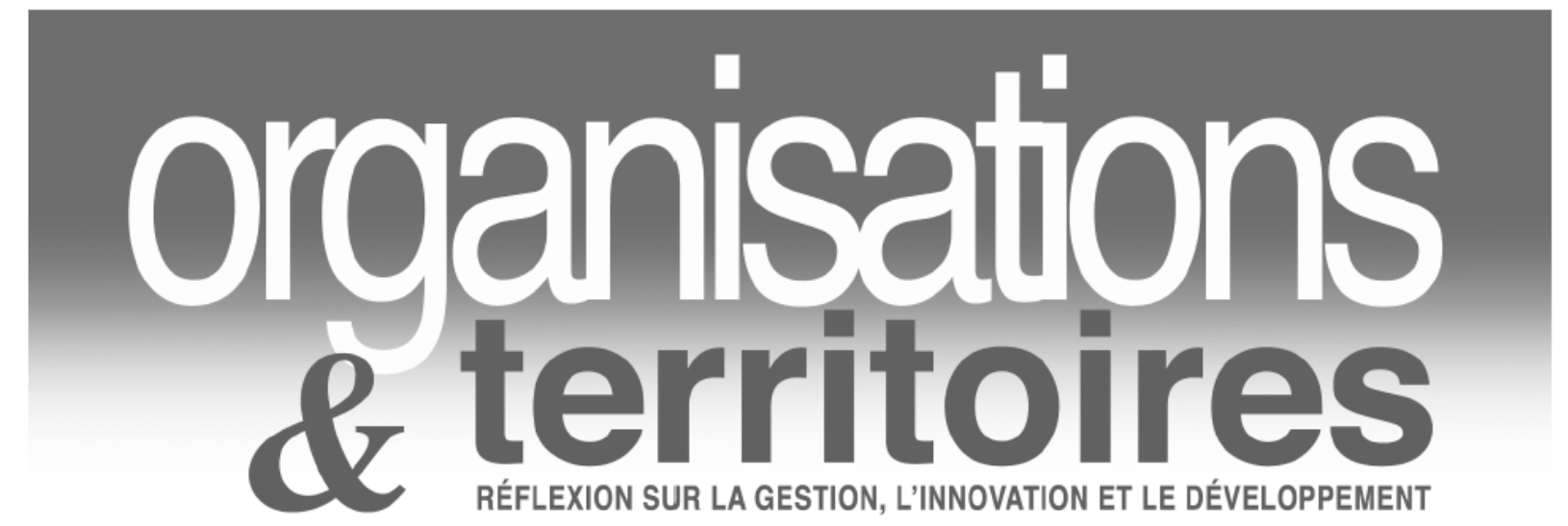

\section{Site Web}

\section{WwW.uqac.ca/revueot}

www.uqac.ca/revueot

www.uqac.ca/revueot

\section{www.uqac.ca/revueot}

Nous vous invitons à l'explorer et à nous faire parvenir vos commentaires et suggestions afin d'en améliorer le contenu et la présentation. 